\title{
Brote inusitado de leishmaniasis cutánea en zona rural de Ibagué: desafíos de la notificación
}

\section{Unusual outbreak of cutaneous leishmaniasis in rural Ibagué: notification challenges}

\author{
Blanca Lisseth Guzmán-Barragán ; Catalina Ballesteros-González; Daniela Torres-González; Yessica Lorena Guzmán ${ }^{4}$
}

\begin{abstract}
1MVZ, Ph.D. Universidad de Ciencias Aplicadas y Ambientales, U.D.C.A, Grupo Ciencia Animal. Bogotá, D.C., Colombia; e-mail: blancalissethguz@hotmail. com; (D) https://orcid.org/0000-0002-3465-1114

Estudiante de MV. Universidad de Ciencias Aplicadas y Ambientales, U.D.C.A, Semillero de Epidemiologia y Salud Pública Veterinaria. Bogotá, D.C., Colombia; e-mail: anballesteros@udca.edu.co; (iD) https://orcid.org/0000-0003-1603-4435

${ }^{3}$ Estudiante de MV. Universidad de Ciencias Aplicadas y Ambientales, U.D.C.A, Semillero de Epidemiologia y Salud Pública Veterinaria. Bogotá, D.C., Colombia; e-mail: danielatorres@udca.edu.co; (D) https://orcid.org/0000-0001-9017-4589
\end{abstract}

${ }^{4}$ MVZ. Unidad de Salud de Ibagué, Grupo Ibagué Saludable. Ibagué - Tolima, Colombia; e-mail: ylguzman@gmail.com; (D) https://orcid.org/0000-0002$2232-3826$

Cómo citar: Guzmán-Barragán, B.L.; Ballesteros-González, C.; Torres-González, D.; Guzmán, Y.L. 2021. Brote inusitado de leishmaniasis cutánea en zona rural de Ibagué: desafíos de la notificación. Rev. U.D.C.A Act. \& Div. Cient. 24(1):e1502. http://doi.org/10.31910/rudca. v24.n1.2021.1502

Artículo de acceso abierto publicado por Revista U.D.C.A Actualidad \& Divulgación Científica, bajo una Licencia Creative Commons CC BY-NC 4.0

Publicación oficial de la Universidad de Ciencias Aplicadas y Ambientales U.D.C.A, Institución de Educación Superior Acreditada de Alta Calidad por el Ministerio de Educación Nacional.

Recibido: marzo 17 de $2020 \quad$ Aceptado: enero 20 de $2021 \quad$ Editado por: Ingeborg Zenner de Polanía

\section{INTRODUCCIÓN}

La leishmaniasis es una infección de amplia distribución mundial. Es causada por un protozoario del género Leishmania, que incluye más de 20 especies (Torres et al. 2017). La leishmaniasis presenta tres formas clínicas: cutánea, con presentación de ulcera cutáneas; mucosa, afectando la mucosa del tabique nasal, paladar y faringe y visceral, afectando órganos internos y la médula ósea casi siempre fatal (Handler et al. 2015). Esta enfermedad es transmitida por un insecto vector del género Lutromyia (Diptera: Psychodidae) y los animales domésticos y animales silvestres pueden actuar como reservorios, aumentando el potencial de transmisión (Calderón et al. 2018). En zonas endémicas, la enfermedad es asociadas con las condiciones de pobreza, falta de saneamiento básico, malas condiciones de viviendas, malnutrición, desplazamiento de la población, condiciones higiénico-sanitarias, el sistema inmunitario (Alvar et al. 2006).

La OMS estima 12 millones de personas en el mundo infectadas por leishmaniasis y 350 millones más, en riesgo de infectarse; cada año ocurren alrededor de 0,9 y 1,6 millones de casos nuevos de leishmaniasis en el mundo, causando entre 20.000 a 30.000 muertes por año (WHO, 2019). La leishmaniasis cutánea es la forma más frecuente; en el mundo, se reportan entre 600.000 y 1 millón casos nuevos al año, principalmente, en las Américas, la cuenca del Mediterráneo, Oriente Medio y Asia Central (WHO, 2019). En América Latina, entre el periodo de 2001-2017, se reportaron un total de 940.396 casos nuevos de leishmaniasis cutánea y mucosa, provenientes de 17 de los 18 países endémicos; los países con mayor reporte son Brasil seguido de Colombia, Perú y Nicaragua (OPS, 2019; Karimkhani 
et al. 2016). En Colombia, entre el 2008 a 2018, se han notificado 110.366 casos, en sus tres formas clínicas; el 98,50\% de ellos corresponden a leishmaniasis cutánea (INS, 2018).

La leishmaniasis presenta desafíos para su control por su diversidad etiológica, compleja epidemiologia, dificultades diagnósticas y falta de herramientas para un adecuado y oportuno tratamiento (Antinori et al. 2012; Alvar et al. 2006). Un objetivo frente a las enfermedades infecciosas desatendidas (EID) y al plan de acción de Leishmaniasis es la disminución de la letalidad de leishmaniasis visceral y la proporción de casos de leishmaniasis cutánea en los $\leq 10$ años en el mundo (OPS, 2019). El objetivo de este estudio fue describir el brote de leishmaniasis cutánea inusitado ocurrido en la zona rural del municipio de Ibagué, en el 2017.

\section{MATERIALES Y MÉTODOS}

El brote de leishmaniasis cutánea ocurrió en veredas del municipio de Ibagué, ubicado en el centro-occidente de Colombia, en la región andina, coordenadas geográficas $4^{\circ} 26^{\prime} 16^{\prime \prime} \mathrm{N}$ y $75^{\circ} 12^{\prime} 02^{\prime \prime} \mathrm{O}$, temperatura anual entre 25 a $28^{\circ} \mathrm{C}$, a una altitud de 1.250 y $1.400 \mathrm{~m}$ s.n.m., las precipitaciones fluctúan entre 2.000 y $3.000 \mathrm{~mm}$ anuales. Las veredas afectadas fueron Honduras, con 133 habitantes; San Cristóbal, 214; San Francisco, 268 habitantes; Caucho Parte Alta, con 71; Charo Rico, 258, pertenecientes a la cuenca mayor del río Combeima, subcuenca del río Coello, con un clima tropical boscoso. El análisis del brote fue elaborado a partir de información epidemiológica reportada en documentos, reportes, encuestas, comités y diagnósticos. Se realizó un análisis descriptivo de los casos, considerando el tiempo y el espacio, las características sociodemográficas, las características clínicas, el diagnóstico, el tratamiento, la evolución de las lesiones y las medidas de control y prevención.

El diagnóstico, se realizó mediante análisis parasitológicos e histopatológicos en el laboratorio de parasitología de la USI E.S.E, a partir de muestras tomadas de los pacientes, con lesiones activas. Técnicas moleculares fueron realizada en un paciente de 60 años, la muestra fue enviada y procesada por el laboratorio de parasitológica de la Universidad Nacional de Colombia, mediante: (a) reacción en cadena de la polimerasa (PCR) gen ribosomal de Leishmania spp.18S; (b) reacción en cadena de la polimerasa (PCR) gen de Leishmania spp. Hsp 70 y (c) restricción con enzimas Haelll y Bccl. Para el estudio entomológico, se instalaron trampas de capturas de insectos de CDC de luz incandescente en el intradomicilio, peridomicilio y extradomicilio de las viviendas de las veredas Cauchos Parte Alta, Charco Rico y Honduras. Se georreferenció cada vivienda, se registraron los dados de altitud, temperatura y la humedad relativa. La identificación taxonómica de los vectores, se realizó en el laboratorio departamental de salud pública.

Se llevó a cabo una caracterización socioambiental, mediante una encuesta semiestructurada, aplicada a todas las 226 viviendas de las veredas afectadas y un total de 1.072 personas, aplicada por los técnicos de saneamiento básico de la Secretaría de Salud y la USI E.S.E., con previo consentimiento informado. Los resultados de la encuesta fueron sistematizados y analizados descriptivamente.

\section{RESULTADOS Y DISCUSIÓN}

El brote inició el 30 de mayo de 2017, con un total de 39 casos, entre la semana 17 a la 51, del 2017; los casos pertenecen a la vereda San Cristóbal, 48,7\%; Honduras, 20,5\%; San Francisco, 20,5\%; Charco Rico, 7,7\% y Cauchos Parte Alta, 2,6\% (Figura 1). La región Andina es la que presenta mayor número de casos de leishmaniasis cutánea; entre el 2009 a 2010, se alcanzó un total de 12.847 casos, mostrando un aumento significativo de la incidencia en el departamento del Tolima (Herrera et al. 2018). La caracterización demográfica de los casos muestra que el 56,4\% fueron del género masculino y 43,6\%, femenino. Los grupos de edad más afectados son los comprendidos entre el 35 a 39 años, con el 23,1\% y de 40 a 44, de 15,4\%, edad con mayor actividad laboral; el 33,4\% fueron menores de 19 años, siendo registrados casos en menores de 5 años. La principal actividad económica fue agricultores, $51,2 \%$, seguido de ama de casa, $28,2 \%$ y $20,6 \%$, otros. $\mathrm{El} 79,5 \%$ de los casos perteneces al régimen subsidiado y el 15,4\%, al contributivo; el 5,1\% no presenta una afiliación en el sistema de salud.

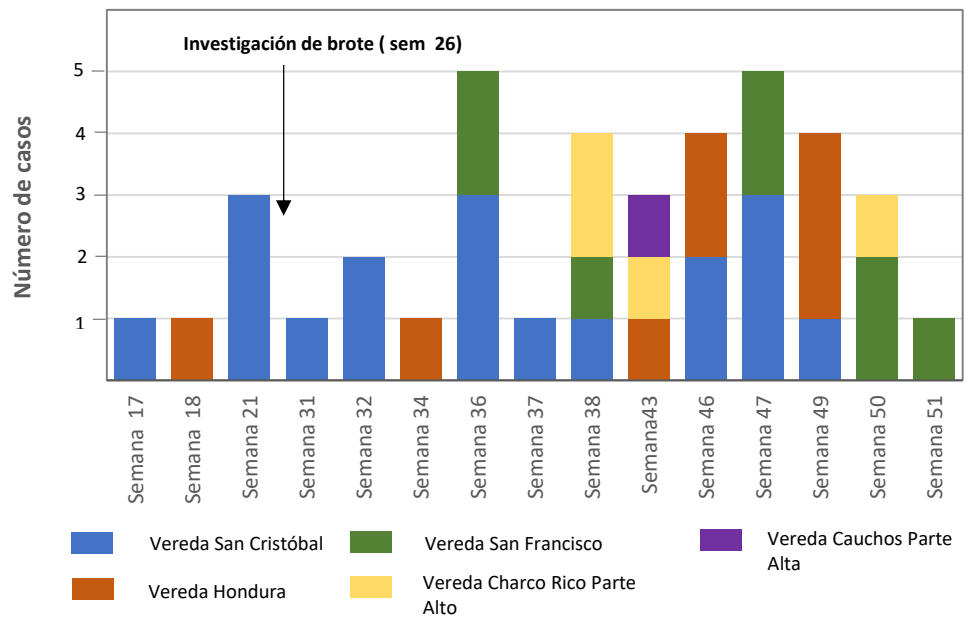

Figura 1. Curva epidémica del brote de leishmaniasis cutánea en zona rural de Ibagué, 2017. 
Las lesiones comenzaron en el sitio de la picadura: el 33,3\%, piernas; $20 \%$, brazos; $8,9 \%$, espalda; $6,7 \%$, abdomen; $4,4 \%$, cara y $2,2 \%$, orejas (Figura 2). El 28,2\% de los pacientes refiere tener menos de un mes de evolución de la lesión; $38,5 \%$ refiere un mes de evolución; 20,5\%, 2 meses y 12,8\%, 3 meses. Se realizó el tratamiento a 26 pacientes con glucantime inyectable (Antimoniato de meglumina), a los pacientes menores de edad y a los que presentaban complicaciones cardiacas y renales ( 3 pacientes), se les suministro miltefosina oral o anfotericina B liposomal. Los principales efectos adversos fueron dolores osteomusculares, malestar general y fiebre; ningún tratamiento fue suspendido. Se evidencia amplias distancias para acceder al tratamiento, puesto que los pacientes recorren entre 10,2 a 20,3km desde las veredas hasta la USI E.S.E., ubicada en el casco urbano; los resultados evidencian una subnotificación de casos. En Colombia, la transición al actual modelo de salud ha centralizado la asistencia en salud en los cascos urbanos, dificultando la asistencia sanitaria en la zona rural (Vargas et al. 2010).

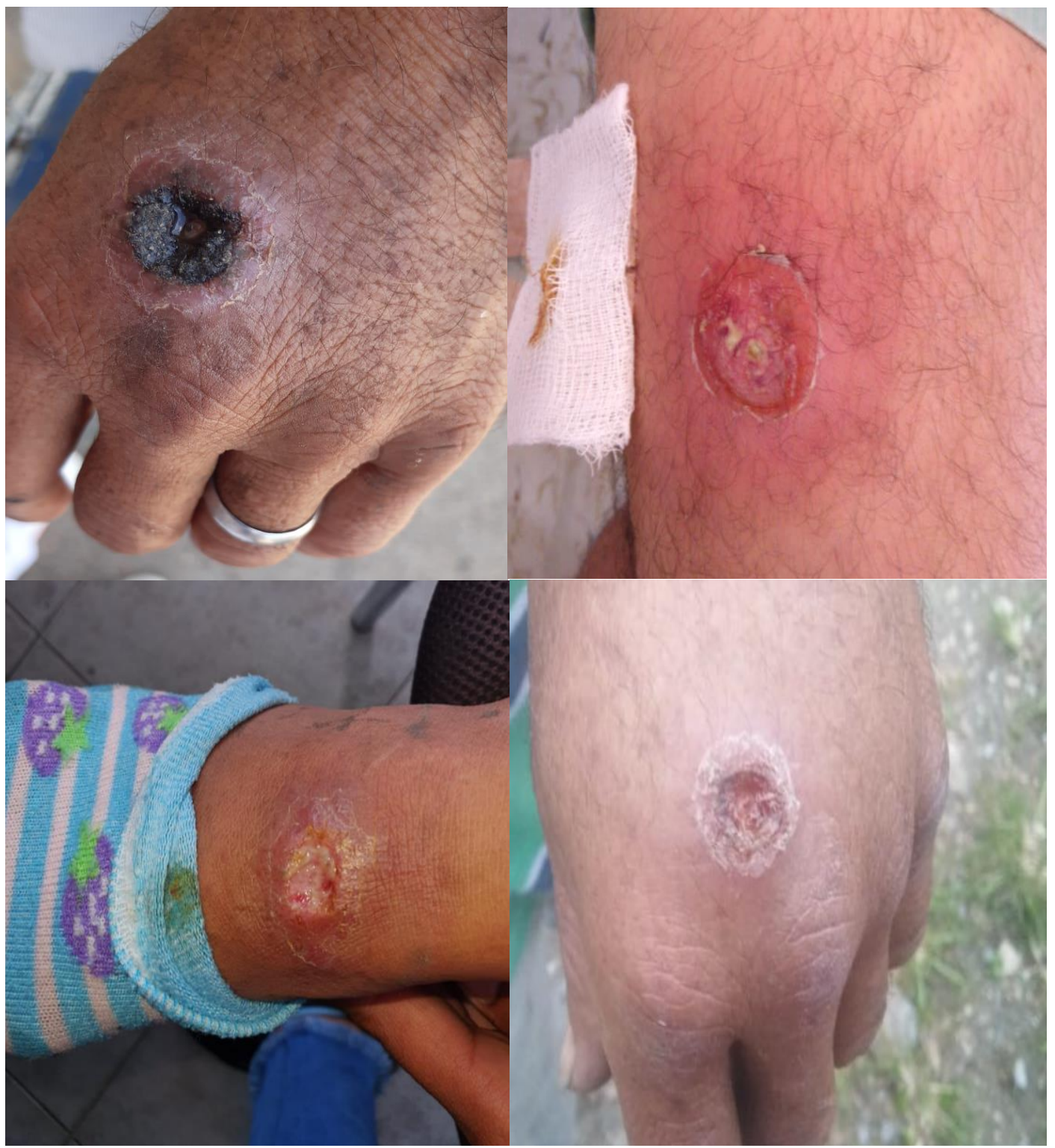

Figura 2. Lesiones de los casos de Leishmaniasis cutánea en el brote ocurrido en zona rural de Ibagué, 2017. Fuente: Secretaría de Salud de Ibagué.

El diagnóstico molecular identificó la especie Leishmania (Vannia) guyanensis. En Colombia, se ha registrado a L. guyanensis en la región Andina, Caribe, Amazonas y Orinoquia (Salgado-Almario et al. 2019); recientemente, se reportó en la región Pacífica (Martínez et al. 2021). Su amplia distribución puede estar relacionada a desplazamiento, causado por el conflicto interno, la migración de los reservorios y la participación de nuevos actores epidemiológicos (Martínez et al. 2021). El diagnóstico de la especie es necesario para 
establecer tratamientos más adecuados, considerando la variabilidad en la sensibilidad en el tratamiento. La L. guyanensis es menos sensible a los antimoniales pentavalentes (Arevalo et al. 2007); en Manaos (Brasil), se demostró que la miltefosina era más efectiva que el antimonial pentavalente, para obtener la mejoría de las lesiones de Leishmaniasis cutánea (Chrusciak-Talhari et al. 2011). En Colombia, se requiere fortalecer el diagnóstico en los casos reportados, en el vector y para la identificación de reservorio.

Se recolectaron un total de 106 flebótomos: 14,1\% (15), en domicilio; 32,1\% (34), peridomicilio y el 53,8\% (57), en extradomicilio, pertenecientes a la especie de Lutromyia longiflocosa. A nivel nacional es la segunda especie con mayores registros, después de la L. gomerzi; su distribución en el país es amplia, principalmente, en la región del Caribe, el Pacífico y los Andes (Ferro et al. 2015). Se observa un aumento de la distribución geográfica y de altitud, considerando que las veredas colindan con el municipio endémico de Rovira, ubicado a $870 \mathrm{~mm}$ y las veredas, entre $1.556 \mathrm{~mm}-1.846 \mathrm{~mm}$ de altitud, con una distancia de $12,3 \mathrm{~km}$ a $15,9 \mathrm{~km}$, entre las veredas y el municipio de Rovira. Peterson et al. (2017) sugieren la transmisión intradomiciliaria a partir de la presencia de L. longipalpis en el domicilio; la ampliación geográfica de L longipalpis ha sido asociados a factores ambientales, desforestación, transformación de los ecosistemas, cambios en la temperatura resultado del cambio climático o la transformación de los ecosistemas.

El levantamiento socioambiental mostró condiciones favorables para la presencia de la Lutzomia spp.; en el 65,1\% de las viviendas, las paredes son de ladrillo y cemento; el $24,7 \%$, de adobe y bahareque y un $10,2 \%$, en madera. El $86,2 \%$, los techos son de eternit y el $30,1 \%$ presenta piso de tierra. La disposición de basuras (54,8\%), la realiza a campo abierto; $34,9 \%$, quemadas y $10,3 \%$, enterradas. El 65,1\% posee inodoro en las viviendas y el 100\% de los hogares no cuenta con suministro de agua tratada. Las viviendas, en su mayoría, cuentan con gallineros y cocheras y presencia de animales domésticos. Asimismo, los pobladores, en su mayoría, se dedican a la caficultura, ambiente adecuado para el vector y su reproducción (Alexander et al. 2001).

Entre las acciones de prevención y de control, se realizó aplicación de insecticida, promoción de prácticas de protección personal y entrega de toldillos y repelentes, así como el fortalecimiento de las capacidades de respuesta de los actores del SGSS. Los resultados de la encuesta muestran que el 18,5\% utilizaban algún tipo de implementos contra mosquitos; el $74,5 \%$ reporta la ausencia e intermitencia de los programas de salud pública y el 82,3\% manifiestan desconocer la enfermedad.

Debilidades de los programas de salud pública en Colombia, como la descentralización parcial, procesos de contratación, prácticas clientelistas, capacidad técnica, falta de personal idóneo, inestabilidad financiera de las instituciones, pueden influenciar la implementación de programas de prevención de la Leishmaniasis (Marín et al. 2016).

\section{REFERENCIAS}

1. ALEXANDER, B.; AGUDELO, L.A.; NAVARRO, F.; RUIZ, F.; MOLINA, J.; AGUILERA, G.; QUIÑONES, M.L. 2001. Phlebotomine sandflies and leishmaniasis risks in Colombian coffee plantations under two systems of cultivation. Med and Vet Entomol (United Kingdom). 15(4):364373.

https://doi.org/10.1046/j.0269-283x.2001.00322.x

2. ALVAR, J.; YACTAYO, S.; BERN, C. 2006. Leishmaniasis and poverty. Trends Parasitol. 22(12):552-557. https://doi.org/10.1016/j.pt.2006.09.004

3. ANTINORI, S.; SCHIFANELLA, L.; CORBELLINO, M. 2012. Leishmaniasis: new insights from an old and neglected disease. Eur. J. Clin. Microbiol. Infect. Dis. (Alemania). 31(2):109-118. https://doi.org/10.1007/s10096-011-1276-0

4. AREVALO, J.; RAMIREZ, L.; ADAUI, V.; ZIMIC, M.; TULLIANO, G.; MIRANDA-VERASTEGUI, M.; LOZANO, M.; LOAYZA-MURO, R.; DE DONCKER, S.; MAURER, A.; CHAPPUIS, F.; DUJARDIN, J.; LLANOS-CUENTAS, A. 2007. Influence of Leishmania (Viannia) species on the response to antimonial treatment in patients with American tegumentary leish maniasis. J. Infect. Dis. (EE.UU). 195(12):184-651.

https://doi.org/10.1086/518041

5. CALDERÓN-ANYOSA, R.; GAVLEZ-PETZOLDT, C.; GARCIA, P.; CARCAMO, C. 2018. Housing characteristics and Leishmaniasis: A Systematic Review. Am. J. Trop. Med. Hyg. (EE.UU). 99(6):1547-1554. https://doi.org/10.4269/ajtmh.18-0037

6. CHRUSCIAK-TALHARI, A.; DIETZE, R.; CHRUSCIAK-TALHARI, C.; DA SILVA, R.; GADELHA, Y.; DE OLIVEIRA, P.; LIMA, P.; TALHARI, S. 2011. Randomized controlled clinical trial to access efficacy and safety of miltefosine in the treatment of cutaneous leishmaniasis caused by Leishmania (Viannia) guyanensis in Manaus. Am. J. Trop. Med. Hyg (EE.UU). 84(2):255-260. https://doi.org/10.4269/ajtmh.2011.10-0155

7. FERRO, C.; LOPEZ, M.; FUYA, P.; LUGO, L.; CORDOVEZ, J.; GONZÁLEZ, C. 2015. Spatial distribution of sand fly vectors and eco-epidemiology of cutaneous leishmaniasis transmission in Colombia. PloS one. 10(10). https://doi.org/10.1371/journal.pone.0139391

8. HANDLER, M.; PATEL, P.; KAPILA, R.; Al-QUBATI, Y.; SCHWARTZ, R. 2015. Cutaneous and mucocutaneous leishmaniasis. J. Am. Acad Dermatol. (EE.UU). 73(6):897908.

https://doi.org/10.1016/j.jaad.2014.08.051 
9. HERRERA, G.; THERAN, A.; PRADILLA, I.; VERA, M.; RAMIREZ, J.D. 2018. Geospatial-temporal distribution of tegumentary Leishmaniasis in Colombia (2007-2016). PLOS Negl Trop Dis (EE.UU). 12(4):e0006419. https://doi.org/10.1371/journal.pntd.0006419

10. INSTITUTO NACIONAL DE SALUD, INS. 2018. LEISHMANIASIS CUTÁNEA, MUCOSA Y VISCERAL. Instituto nacional de salud. INS. Disponible desde Internet en: https://www.ins.gov.co/buscadoreventos/Informesdeevento/LEISHMANIASIS_2018.pdf (con acceso $3 / 11 / 18)$

11. KARIMKHANI, C.; WANGA, V.; COFFENG, L.E.; NAGHAVI, P.; DELLAVALLE, R.P.; NAGHAVI, M. 2016. Global burden of cutaneous leishmaniasis: a cross-sectional analysis from the Global Burden of Disease Study 2013. Lancet Infect Dis (EE:UU). 16(5):584-591. https://doi.org/10.1016/S1473-3099(16)00003-7

12. MARÍN, G.M.; RAMIREZ, A.; RUIZ, A. 2016. Tensiones en las decisiones en salud pública en el sistema de salud colombiano: el bien común en confrontación con los intereses y prácticas particulares. Universidad de Antioquia. Facultad Nacional de Salud Pública "Héctor Abad Gómez" (Medellín). 504p.

13. MARTÍNEZ ABAD, L.P.; PATERNINA, L.E.; PATERNINA-GÓMEZ, M.; PÉREZ DORIA, A.; BEJARANO MARTÍNEZ, E.E. 2021. Un nuevo registro de Leishmania (Viannia) guyanensis (Trypanosomatidae) de la costa pacífica de Colombia. Acta Biol. Colomb. 26(1). http://dx.doi.org/10.15446/abc.v26n1.78321

14. ORGANIZACIÓN PANAMERICANA DE LA SALUD, OPS. 2019. Leishmaniasis Informe epidemiológico de las Américas, marzo de 2019. Disponible desde Internet en: http://iris.paho.org/xmlui/handle/123456789/50505 (con acceso 13/09/2020).
15. PETERSON, A.T.; CAMPBELL, L.P.; MOO-LLANES, D.A.; TRAVI, B.; GONZALEZ, C.; FERRO, M.C.; FERREIRA, G.E.M.; BRANDAO-FILHO, S.P.; CUPOLILLO, E.; RAMSEY, J.; LAFFER, A.M.C.; PECH-MAY, A.; SHAW, J.J. 2017. Influences of climate change on the potential distribution of Lutromyia longipalpis sensu lato (Psychodidae: Phlebotominae). Int. J. Parasitol. (Netherlands). 47(1011):667-674. https://doi.org/10.1016/j.ijpara.2017.04.007

16. SALGADO-ALMARIO, J.; HERNANDEZ, C.; OVALLE, C. 2019. Distribución geográfica de las especies de Leishmania en Colombia, 1985-2017. Biomedica. 39(2):78-90. https://doi.org/10.7705/biomedica.v39i3.4312

17. TORRES-GUERRERO, E.; QUINTANILLA-CEDILLO, M.; RUIZ-ESMENJAUD, J.; ARENAS, R. 2017. Leishmaniasis: a review. F1000Res (United Kingdom). 26(6):750. https://doi.org/10.12688/f1000research.11120.1

18. VARGAS-LORENZO, I.; VAZQUEZ-NAVARRETE, M.; MOGOLLON-PEREZ, A. 2010. Acceso a la atención en la salud en Colombia. Rev Salud Pública. (Colombia). 12(5):701-712. https://doi.org/10.1590/S0124-00642010000500001

19. WORD HEALTH ORGANIZATION, WHO. 2019. Leishmaniasis en las Américas - Hoja informativa para los trabajadores de salud. WHO; Washington. Disponible desde Internet en: https://www.paho.org/hq/index.php?option=com content\&view $=$ article $\& i d=13648: 1$ eishmania sis-fact-sheet-health-workers\&Itemid $=40721 \&$ lang $=\mathrm{es}$ 